\title{
A Method for Automatically Detecting the Living-Alone Elderly's Abnormal Behavior with RTLS
}

\author{
Junshuai Liang \\ Department of Information Engineering \\ Qingdao University \\ Qingdao, China \\ liangjunshuai@foxmail.com
}

\author{
Zhongqing Yu, Shuai Yang \\ Department of Information Engineering \\ Qingdao University \\ Qingdao, China \\ yu@haiersoft.com/qduxgys@sina.com
}

\begin{abstract}
With the development of society, the population of aging has become increasingly prominent. Especially children do not live together with the elderly, when tumbles and other dangerous situations happen to the elderly who live alone in their own room, and their lives may be threatened for lack of timely treatment. In this paper, we have discovered a solution to monitor the elderly by means of equipping their home with the indoor positioning system which is based on the UWB technique and its precision between $10 \mathrm{~cm}$ and $30 \mathrm{~cm}$. Experiments show that the rate of false alarm is relatively low, and it can be accepted that using the solution which is provided in this paper to send alert when checking the elderly's abnormal behavior.
\end{abstract}

Keywords-assisted living environment; abnormal behavior detection; threshold value; classification; the Living-Alone Elderly

\section{INTRODUCTION}

Abnormal behavior detection can be used in many fields such as surveillance systems, network intrusion detection, and healthcare monitoring systems. Especially, in healthcare monitoring systems, most of the research has used images from video cameras and analyzed them with image related techniques [1, 2]. However, the elderly or patients usually do not want to be monitored by the means of video cameras, because of privacy violation issues. Therefore, we propose a human behavior detection system that does not depend on images from video camera, but uses noninvasive wireless sensor techniques.

The purpose of our work is to determine whether an episode taking place is normal or not by thethreshold of duration that the elderly stay in monitored areas. If the duration that the elderly stay in monitored area exceeds the threshold, then the system makes a warning so that it can catch caregiver's interest.

\section{RELATED WORK}

Yin et al. present a novel two-phase approach to detectabnormal activities based on wireless sensors attached to a human body in [3]. They present an automated methodology for extracting the spatiotemporal activity model of a person using a wireless sensor network deployed inside home in [4]. Lühr et al. propose a novel application of transaction association rule (IAR) mining to detect anomalous behavior in smart home occupants in [5]. Krishnan et al. propose and evaluate a sliding window which is based an approach to perform activity recognition in an online or streaming fashion, recognizing activities as and when new sensor events are recorded in [6].

There are several previous works that used Clustering techniques in the application of recognition of human being's behavior or healthcare monitoring systems especially in tracking data analyzing in [7]. They build a prototype system to confirm the importance, feasibility and validity of the modal. Rodriguez proposed an approach based on the idea that cluster centers are characterized by a higher density than their neighbors and by a relatively large distance from points with higher densities in [8]. Miao et al. introduce the concept of intelligent assistant, and explores an activity model based on the regulations of the elderly and similarity of situations to detect outlier in [9]. Chen et al. propose D-Stream, a framework for clustering stream data using a density-based approach in [10].

In the area of human behavior recognition, this paper focus on the duration the elderly stay in monitored areas and get monitored areas which we are interested after analyzing these data by a clustering method which is based on the DBSCAN algorithm. There are three aspects to establish the system for automatic detection of the living-alone elderly's abnormal behavior. First, based on location data of the elderly's daily life, we get the room zoning after analyzing these data by a clustering method which is based on density. The DBSCAN algorithm is a classic density-based clustering algorithm. It can automatically determine the number of clusters and arbitrary shapes of clusters. In the clustering process of the algorithm, there are two parameters, Eps and minPts, have to be specified by user. We offer a method which can determine the two parameters automatically via analysis of the statistical characteristics of the dataset, which enabled clustering process of DBSCAN fully automated. Experimental results indicate that it can get appropriate parameters and gain a rather high validity of clustering through the DBSCAN algorithm. The paper focuses on the method how to get monitored areas which are used to monitor human behavior by the DBSCAN algorithm, and how to get the two parameters of the algorithm automatically.

\section{DEFINITIONS}

This section focuses on how to build models about the process of abnormal behavior. We set the model of interested 
area in section 3.1, abnormal behavior detection rule model in section 3.2 and user model in section 3.3.

\section{A. Interested hotspot model}

The elderly's daily activities can be represented by the triples: theinterested hotspot ID, the timestamp when the elderly enter the monitored area and the duration the elderly stay in this area. We let hotspot ${ }_{i}$ be an event which the elderly has stayed at this monitor area, where $i$ indicate the order of monitored area which the elderly has stayed in.

$$
\operatorname{hotspot}_{i}=(S, T, D)
$$

Where $\mathrm{S}$ is an interested hotspot ID that can represent the location area where the elderly have stayed, $\mathrm{T}$ is a time stamp when the elderly will be entering the monitored area and $\mathrm{D}$ is a duration, which is two timestamps difference between when the elderly enter and when the elderly leave. For example, we can regard the tripe (Kitchen,1200,40) as an event which can be used to be expressed that the elderly have stayed in kitchen about 40 minutes when the elderly enter at 12:00.

\section{B. Abnormal behavior detection rule model}

We let $r u l e_{i}$ be an rule which is used to be checked whether or not the duration the elderly stay in monitored area (S) at a certain period of time (I) exceed the set duration threshold (Dt).

$$
\text { rule }_{i}=(S, I, D t)
$$

Where $\mathrm{S}$ is the id of the interested hotspotthat can represent the location area where the elderly have stayed, I represent the index of the intervalwhich is divided by an hour a day and Dt is a duration threshold which is set according to statistical data the elderly stay in monitored area (S) at a certain period of time (I).Forinstance,the triple (Kitchen,11,45) can indicatethat it isn't longer than 45 minutes when the elderly stay in kitchen at the 11th period a day (12:00 13:00).

\section{ABNORMAL BEHAVIOR DETECTION}

In this section, we describe the process of the monitoring system which can detect abnormal human behavior, the method to get interested hotspot, the method to get the parameters of the DBSCAN algorithm automatically and the method to build an alert line according to statistical data the elderly stay in monitored area at a certain period of time.

\section{A. The positioning system}

The longer the duration that the elderly stay in the monitored area is, the more likely abnormal behaviors occur in these sensitive areas and if it can't get timely alarm, there may be life-threatening to the elderly. So we monitor these hot spots that it can be used to send alerts to the elderly's relatives and health professionals. We have to use UWB in our RTLS because of the accuracy of indoor position and its accuracy in positioning can be controlled in 10 centimeters while the cost is high.

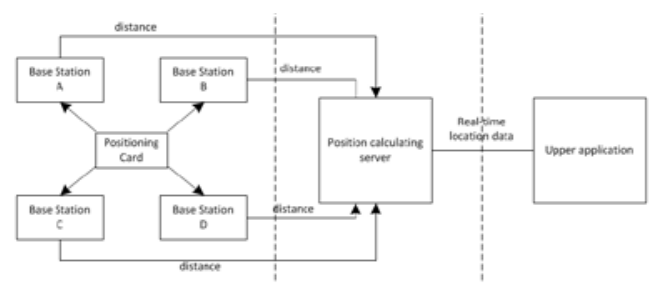

Fig. 1. The principle diagram of indoor positioning systems

In Fig. 1, the system consists of four base stations which collect the data of distance between cards which the elderly should hold and base station. We get the real-time location data from server which can calculate the distance data between base station and positioning card according to the localization algorithm. A triple can be used to explain that the elderly's indoor position in a moment. Where $T_{S}$ is a timestamp, $\mathrm{x}$ and y can represent the location where the elderly stay.

\section{B. Interested hotspots}

These hot spots can be generated by the DBSCAN algorithm through these location data. The DBSCAN algorithm is a density-based clustering algorithm, and its two parameters of the DBSCAN algorithm will be set manually in advance. In this paper a method to get Eps and minPts will be proposed. Due to time rush and limited conditions, the characteristics of the DBSCAN algorithm can't be tested in actual environment and some trajectory data are simulated in Fig. 2. The efficiency of the algorithm is evaluated by using the method of calculating the size of the algorithm, and the effect of the cluster. In this paper, the distance between two points is based on the density-based clustering algorithm, which is based on the distance between two points in Manhattan distance.

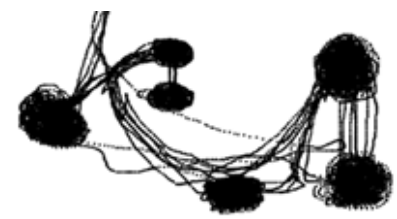

Fig. 2. The indoor simulation trajectory data

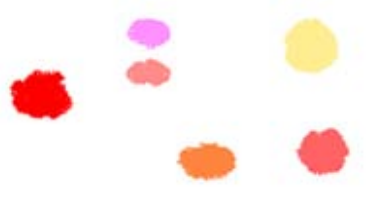

Fig. 3. The distribution after clustering

By setting Eps and minPts required by the DBSCAN algorithm, the clustering effect is better when the Eps value is 11 and the minPts value is 120 , which is shown in Fig. 3. 


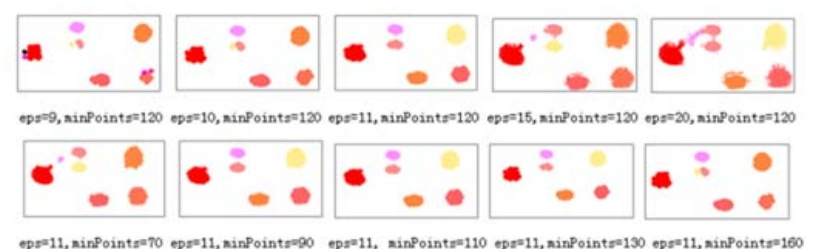

Fig. 4. The clustering effect of different Eps and minPts values.

In Fig. 4, the clustering effect is best when Eps is 11 and minPts is 120 in first row. When the number of one point's nearest neighbors is fixed, the smaller EPS is, the less the number of the generated clusters is. It can be determined that the EPS parameters can become a key factor based on density clustering. So, a method to get Eps will be proposed in the following. After the results of the statistical situation shown in Fig. 5, the abscissa represents the distance values of the two points, and the vertical axis represents the number of the equal distance between any two points.

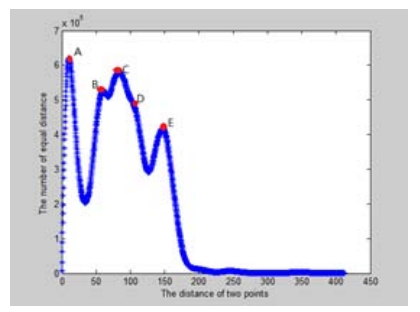

Fig. 5. The distribution of equal distance

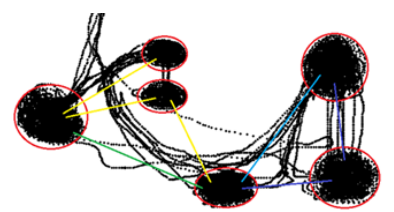

Fig. 6. The distribution of trajectory data

However, there are maximum points in the distribution of the distance in Fig. 5, because of the distance can be gained between any two points in Fig. 6. As is known to all, the first extreme point is the result that we want to get in Fig. 5. When the distance is limited to 50, it can be show in Fig. 7. It can be known that the value of extreme point is about 10 and it matches the value of Eps we use in the DBSCAN algorithm.

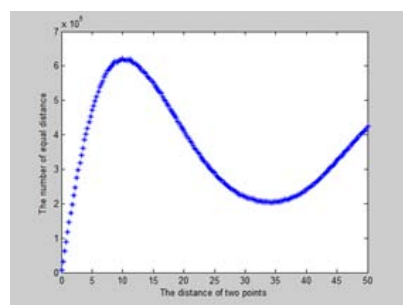

Fig. 7. The distribution of equal distance (distance $<50$ )

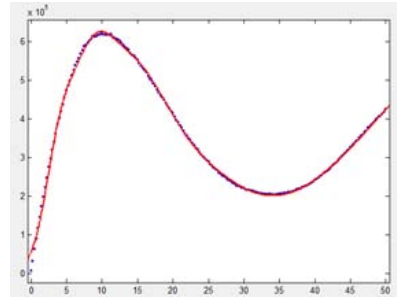

Fig. 8. After fitting the curve by Inverse Gaussian Distribution function

After fitting by inverse Gaussian distribution, the curve is the best. The probability density function of inverse Gauss distribution is shown in formula 3, and can be obtained by maximum likelihood. In order to obtain the value of the extreme point, we need the $f(x)$ derivative. After solving equation $d f(x) / d x=0$, we can get the value of the extreme point $_{x}=\left(3 \mu^{2}+\mu \sqrt{9 \mu^{2}+4 \lambda^{2}}\right) / 2 \lambda$.

$$
f(x)=\left(e^{(x \mu)^{2} /\left(2 x \mu^{2}\right)} \sqrt{\lambda / x^{3}}\right) / \sqrt{2 \pi}
$$

The value of Eps can be obtained from the above discussion, and we can get the number of neighbors of one point by Eps. The value of minPts can be obtained through calculating the expected value of the number of points within the eps range of each point. It can be obtained in formula 4, $m p$ is the value of minPts and $C i$ is the number of neighbors of the point $\mathrm{i}$.

$$
m p=\frac{1}{n} \sum_{i=1}^{n} C_{i}
$$

After the clustering analysis of trajectory data, hot spots should be changed to monitored area in order to monitor the elderly's abnormal behaviors. The position of the center point of the monitored area is generated by calculating the average of points of one cluster in hot spots. The radius is obtained by calculated a half of the longest distance between two points of one cluster. The distribution of indoor partition area can be shown in Fig. 9.

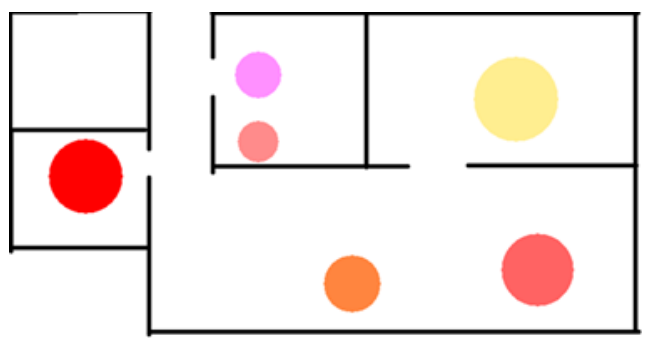

Fig. 9. The distribution of indoor partition area

\section{Generating an alert line.}

As for the elderly's abnormal behavior, this paper mainly according to the rule whether or not the duration of the elderly stay in one monitored area in a certain period in one day can exceed the set duration threshold in advance. If it is true, an alert should be sent to the elderly's relatives and health care provider. The monitored areas can be obtained in section 4.2, and the duration the elderly stay in this area can be obtained by calculating the difference of two time stamps between when the elderly enter and when the elderly leave. It can be shown in Fig. 10 that the distribution of the duration the elderly in four 
monitored areas in one day after calculating the trajectory data which are simulated for the elderly within 240 days.

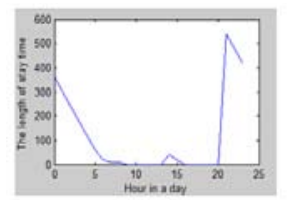

A. bedroom

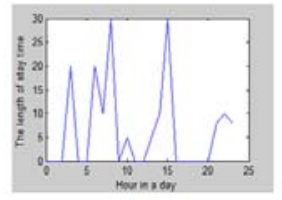

C. bathroom

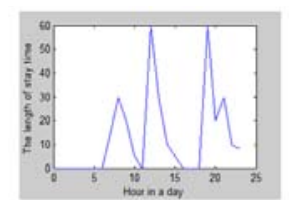

B. kitchen

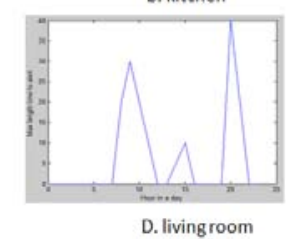

Fig. 10. The duration threshold in four monitored area

In Fig. 10, in part A we can see that the elderly stay longer in the bedroom between $8 \mathrm{pm}$ and 6 am in next day. In part B the duration when the elderly stay in one monitored area less than 30 minutes and it can be inferred that the monitored area is bathroom. We can see from part $\mathrm{C}$ that the elderly stay in this area at $8 \mathrm{am}, 12 \mathrm{am}$ and $7 \mathrm{pm}$ and the duration they stay in this monitored area is close to an hour or more than an hour. It can be inferred that the monitored area is kitchen in part $C$. Compared with part $\mathrm{C}$, it can be inferred that the monitored area in part $\mathrm{D}$ is living room because of the less length of the duration the elderly stay in this monitored area.

\section{EXPERIMENTAL RESULTS}

In this paper trajectory data which are obtained from the elderly's daily life within 210 days are simulated. Monitored areas are mainly used to evaluate accuracy of process which is provided in this paper. At present, there are two kinds of performance standard to evaluate the method to send the alert. One is to ensure the false alarm rate remained at a very low level, and the other one is able to send alert within an acceptable time after getting the elderly's abnormal behaviors. The number of times of total alarm and the number of false alarm during the elderly's 180 days can be shown in table 1 .

TABLE I. THE NUMBER OF TIMES OF ALARM

\begin{tabular}{|l|l|l|}
\hline \multicolumn{1}{|c|}{ 180days } & \multicolumn{1}{c|}{ All Alert Times } & Error Alert Times \\
\hline $0 \sim 60$ & 7 & 1 \\
\hline $0 \sim 120$ & 15 & 2 \\
\hline $0 \sim 180$ & 21 & 4 \\
\hline
\end{tabular}

The number of times of total alarm within 180 days can be shown in table 1 . The rate of false alarm in the first 60 days is 0.14 , and is slightly higher in the first 120 days and 180 days. It may be explained that these alerts added in testing can't match the actual situation. The rate of false alarm is relatively low, and it can be accepted that using the method to send alert when checking the elderly's abnormal behavior.

The threshold of duration that the elderly stay in an area at one period time in one day can be obtained by 90 percentile of values which are statistically derived from historical data. It is shown in Fig. 11 that the different percentile duration threshold that the elderly stay in the bedroom at each period which is divided by an hour in one day. It can be shown that different percentile value of duration threshold can reflect the processing method in the sensitivity of alarm processing.

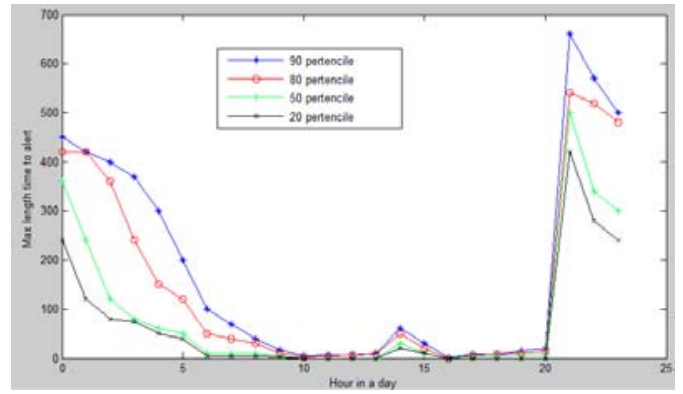

Fig. 11. The sensitivity of the processing method

VI. CONCLUSIONS AND FUTURE WORK

In this paper, a method to get the alert when checking the elderly's abnormal behaviors is proposed. As for the DBSCAN algorithm to get these hotspots, and a method to get Eps and minPts automatically is proposed. After getting these hot spots, we change these to monitored area which we are interested and the alert line which represents the duration threshold that the elderly stay in monitored area at a period. An alert will be sent to the elderly's relatives and health care provider when the duration that they stay in one monitored area can exceed the value of the set threshold in advance.

This paper's purpose is simply getting an alert when checking the elderly's abnormal behavior. It is mainly according to the rule whether or not the duration of the elderly stay in one monitored area in a certain period in one day can exceed the set duration threshold in advance. The reader can estimate the health status of the elderly through calculating the similar between two sequences in these monitored areas.

\section{ACKNOWLEDGEMENTS}

This work was supported by a grant from the Special for Scientific and Technological development of the Construction Cause of Qingdao (No. JK2014-34).

\section{REFERENCES}

[1] Wang Y, Wang D, Chen F, Abnormal behavior detection using trajectory analysis in camera sensor networks, J. International Journal of Distributed Sensor Networks 2014 (2014).

[2] Amin A, Anzum M F, Mondol M H, Abnormal behavior detection of human by video surveillance system, D. BRAC University, 2014.

[3] Yin J, Yang Q, Pan J, Sensor-based abnormal human-activity detection, Knowledge and Data Engineering, IEEE Transactions on, 20(8), 1082-1090.

[4] Crombag E, Plasmeijer I R, Lucas P, Monitoring the elderly using real time location sensing, D. Master's thesis, RADBOUD UNIVERSITY, 2009.

[5] Lühr S, West G, Venkatesh S, Recognition of emergent human behaviour in a smart home: A data mining approach, J. Pervasive and Mobile Computing, 2007, 3(2): 95-116.

[6] Krishnan N C, Cook D J, Activity recognition on streaming sensor data, J. Pervasive and mobile computing, 2014, 10: 138-154.

[7] Ester M, Kriegel H P, Sander J, A density-based algorithm for discovering clusters in large spatial databases with noise, C. Kdd. 1996, 96(34): 226-231.

[8] Rodriguez A, Laio A. Clustering by fast search and find of density peaks, J. Science, 2014, 344(6191): 1492-1496.

[9] MIAO Qiang, ZHOU Xing-she, NI Hong-bo, Research of outlier detection technique based on behavior rule. Computer Engineering and Applications, 2010, 46(15):211-214

[10] Chen Y, Tu L, Density-based clustering for real-time stream data, C. Proceedings of the 13th ACM SIGKDD international conference on Knowledge discovery and data mining. ACM, 2007: 133-142. 